SCIREA Journal of Clinical Medicine

ISSN: 2706-8870

http://www.scirea.org/journal/CM

August 15, 2021

SCIREA

Volume 6, Issue 5, October 2021

\title{
Decoupling of cell refractive index and thickness distribution under three-wavelength phase imaging
}

\author{
Jingrong Liao 1, Yawei Wang ${ }^{2}$, Shuangshuang Xue 2, Yujuan Sun 2, Wen Jiang 2, \\ Yuanyuan $\mathrm{Xu}^{2, *}$ \\ ${ }^{1}$ School of Mechanical Engineering, Jiangsu University, Zhenjiang 212013, China; \\ ${ }^{2}$ School of Physics and Electronic Engineering, Jiangsu University, Zhenjiang 212013, China \\ *Corresponding author: yuanyuanxulark@126.com
}

\begin{abstract}
Decoupling of refractive index (RI) and thickness information of a cell is very importment in cell's analysis. This paper proposes a method for decoupling the refractive index and thickness of a cell under three-wavelength phase imaging. Based on the Quantitative Phase Imaging (QPI) method, the proposed method can obtain the sample's refractive index and thickness distributions by solving three-wavelength phase equations using the approximate setting under dispersion based on the optical and optical dispersion theories. In simulation experiments, the obtained refractive index and thickness distribution results have high accuracy with a maximum error lower than $6 \%$. The validation results show that the method can effectively decouple the refractive index and thickness for homogeneous cells and multimedia non-spherical cells. That demonstrates the method's effectiveness, making up for the shortcomings of traditional methods and playing an important role in $3 \mathrm{D}$ imaging of the cell's sub-structures.
\end{abstract}


Keywords: Quantitative phase imaging; cellular substructure; three-wavelength; decoupling refractive index; thickness distribution.

\section{Introduction}

A cell is the smallest unit of life, and the detection of its structural and morphological characteristics is important in life sciences, analytical chemistry, chemical engineering, information science, bioengineering, medicine, and clinical applications. The cell morphology detection, especially the sub-structural morphology of nucleated cells, is essential for clinical applications. However, most cells, are colorless and transparent, they are difficult to be see with the conventional optical microscopy. To address this issue, a lot of quantitative phase imaging (QPI) techniques have been reported. With quantitative QPI, the phase distribution of a cell can be obtained. In fact, the phase is the product of refractive index and thickness along the axis. For most of cells, they are heterogeneous, so the refractive index and thickness are often coupled in the phase. Therefore, more and more people pay attention to the decoupling of refractive index and thickness.

In digital holographic microscopy of biological cells, the RI and thickness decoupling techniques can be divided into three categories [1]. The first category evaluates the thickness method, including approximate model assessment, direct thickness measurement, and thickness constraint. The second category is an interferometric measurement, and the third category is tomographic phase microscopy (TPM). Approximate model assessment is the most straightforward method, approximating the cell shape as spherical or ellipsoidal, but this method is not applicable to red blood cells. Other methods such as the proposed Lue et al. [2] are based on the Hilbert phase microscope decoupling average RI, achieving thickness confinement by confining living cells in a microfluidic device, with a high acquisition rate and low noise. Kemper [3] introduced a method for measuring the integrated RI of living cells in a suspension using a digital holographic microscope. The Gauss-Newton method was used to solve the nonlinear least square problem to fit the thickness iteratively, and then the RI was obtained. The algorithm analyses the RI of cells without additional sample preparation, and the digital holographic microscope has a multi-focus feature that increases the amount of data collected by simultaneously recording cells in suspension at different focal planes. The direct thickness measurement method measures geometric thickness by different imaging methods, such as atomic force microscopy [4], confocal reflectance 
microscopy [5], and the combination of a confocal fluorescence microscopy and phase microscopy [6], but these methods increase the complexity of the combined system and may damage cells. Tomographic phase microscopy method (TPM) uses the phase [7-11] to image objects from different perspectives. After digitizing the phase map, it is possible to decouple two-dimensional information and obtain a three-dimensional distribution of the RI of the cells, but the method requires multiple measurements and extensive calculations for many angles. The two-interferometry extraction method involves solving two equations and two unknown variables. There are two ways to obtain the two equations. The first is to obtain different refractive indices by putting cells in different environmental fluids [12-14]. Rappaz. [15] proposed a dual-wavelength digital holographic microscopy, that allows to measure the RI and thickness of cells by using the infusion medium dispersion enhanced by extracellular dyes. This method requires the cells to be exposed to two different surrounding media, increasing the limitations of the cell preparation procedure. It also requires the simultaneous acquisition of dual-wavelength phase images to avoid errors caused by sequential acquisition, especially for studying the dynamic characteristics of samples. The second method is to irradiate cells with two different wavelengths to obtain two different refractive indices [1618], which main advantage is that both measurements can be performed simultaneously, allowing decoupling even for dynamic cells. However, to achieve this, the RI of the environmental medium must be highly dispersive, but this condition is difficult to satisfy. Therefore, Jafard [16] suggested using ethylene glycol (C2H6O2) as the medium, where the difference in RI is 0.02 by changing the wavelength from $532 \mathrm{~nm}$ to $632 \mathrm{~nm}$. However, this solution is toxic to cells, so biological related research and possible dynamic observation may be prohibited. Multi-wavelength phase microscopy refers to two or more different wavelengths irradiating the sample consecutively or simultaneously to obtain two or more phase information. The phase distribution obtained is continuous without unwrapping operations can be used to decouple the RI and thickness of cells.

For simple samples with known dispersion or dispersive media, imaging the sample by two wavelengths may be enough. For example, Peng Guangwei [19] introduced RI decoupling based on dual-wavelength, which assumes that the environment medium is a solution with high dispersion. The two-phase distributions at $532 \mathrm{~nm}$ and $632 \mathrm{~nm}$ wavelengths are used to achieve RI decoupling of heterogeneous mononuclear cells. For red blood cells or other nucleated cells, two wavelengths may not be enough, and multiple wavelengths may be required to image them without the use of high dispersion media. 
Considering these methods said above are difficult to decouple for substructure cells with any shape. This paper proposes a method for decoupling the RI and determining the thickness distribution under three-wavelength phase microscopic imaging to address the former decoupling difficulties. In the method, the Cauchy dispersion law is used to ignore the parameters of higher power terms. According to the relationship between phase and optical path difference, the refraction index of cell cytoplasm and nucleus can be obtained by mathematical analysis, and the corresponding thickness distribution can be further obtained. The method can be applied to spherical or non-spherical cells and to any dispersive medium. Therefore, this method is suitable for decoupling the RI of substructure cells with any shape and allows the thickness distribution of the cytoplasm and nucleus to be reconstructed, providing an important basis for the analysis of cell morphology.

\section{Method for decoupling thickness and refractive index}

\subsection{Basic theory}

Blood cells are divided into three groups (erythrocytes, leukocytes, platelets) among leucocytes have five subclasses (neutrophils, basophils, eosinophils, monocytes, lymphocytes). Blood cells are usually colorless, spherical, nucleated cells, and the cell cytosol is spherical primarily due to their surface tension and other reasons, and their nucleus is mostly non-spherical. In cell phase microscopy imaging, because the phase contains thickness and RI, there is a coupling between the two physical quantities, which brings difficulties to reconstructing cell 3D morphology imaging. In addition, there is dispersion in the multi-wavelength phase imaging process; that is, the RI of the cells is different at different wavelengths. For normal dispersion, the shorter the wavelength, the greater the RI, and the rate of change of the RI concerning the wavelength is also the dispersion rate $d n / d \lambda<0$. For anomalous dispersion, the longer the wavelength, the greater the RI, and the dispersion rate is greater than 0 , i.e. $d n / d \lambda>0$. The two can be said to be diametrically opposed to each other. In general, the relationship between RI and wavelength can be expressed as:

$$
n_{(\lambda)}=a+b / \lambda^{2}+c / \lambda^{4}
$$


Where $a, b$, and $c$ are three Cauchy dispersion coefficients, which vary based on the varied medium. With the three Cauchy dispersion coefficients, the RI at other wavelengths can be calculated.

In phase microscopy imaging, a multimedium phase body model is used to simulate nucleated cells, as given in Figure 1. Consider the phase image obtained by light along with the Z-axis in the XY plane, and the phase distribution at the three wavelengths is as follows:

$$
\varphi_{i}(x, y)=\frac{2 \pi}{\lambda_{i}}\left[\left(n_{1\left(\lambda_{i}\right)}-n_{0\left(\lambda_{i}\right)}\right) h_{1}(x, y)+\left(n_{2\left(\lambda_{i}\right)}-n_{1\left(\lambda_{i}\right)}\right) h_{2}(x, y)\right]
$$

Where, $i=1,2,3$ corresponds to the three wavelengths, $h_{1}$ is the thickness of the cell (including the thickness of the nucleus), $h_{2}$ is the thickness of the nucleus, $n_{0}, n_{1}$, and $n_{2}$ are the RI of the environment, cytoplasm, and nucleus, respectively.

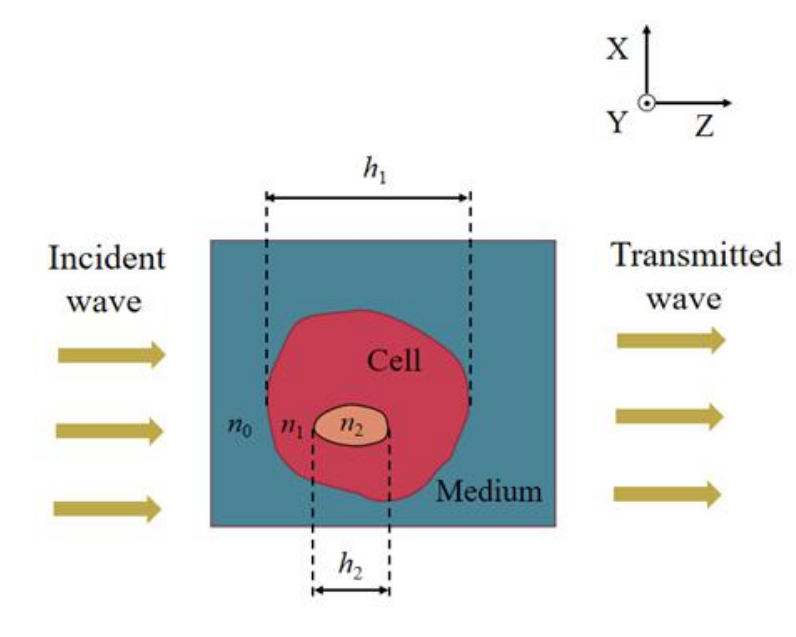

Figure 1. Schematic diagram of the light incident sample.

\subsection{Determination Method of Refractive Index}

Assume that light enters the cell along with Z-axis, ignoring the higher-order term in Eq. (1), i.e., not considering the Cauchy dispersion coefficient $\mathrm{C}$ effect, the phase distribution Eq. (3) can be obtained from Eqs. (1) and (2):

$$
\varphi_{i}(x, y)=\frac{2 \pi}{\lambda_{i}}\left[\left(a_{1}+b_{1} / \lambda_{i}^{3}-a_{0}-b_{0} / \lambda_{i}^{3}\right) h_{1}(x, y)+\left(a_{2}+b_{2} / \lambda_{i}^{3}-a_{1}-b_{1} / \lambda_{i}^{3}\right) h_{2}(x, y)\right]
$$

where $i=1,2,3$.

According to Eq. (3), the phase difference at three wavelengths is as follow, 


$$
\begin{aligned}
\Delta \varphi_{i j}(x, y)= & 2 \pi\left[\left(a_{1}\left(1 / \lambda_{j}-1 / \lambda_{i}\right)+b_{1}\left(1 / \lambda_{j}^{3}-1 / \lambda_{i}^{3}\right)-\right.\right. \\
& \left.a_{0}\left(1 / \lambda_{j}-1 / \lambda_{i}\right)-b_{0}\left(1 / \lambda_{j}^{3}-1 / \lambda_{i}^{3}\right)\right) h_{1}(x, y)+ \\
& \left(a_{2}\left(1 / \lambda_{j}-1 / \lambda_{i}\right)+b_{2}\left(1 / \lambda_{j}^{3}-1 / \lambda_{i}^{3}\right)-\right. \\
& \left.\left.a_{1}\left(1 / \lambda_{j}-1 / \lambda_{i}\right)-b_{1}\left(1 / \lambda_{j}^{3}-1 / \lambda_{i}^{3}\right)\right) h_{2}(x, y)\right]
\end{aligned}
$$

Where $i=1,2,3 ; j=1,2,3 ; i \neq j$. By simplifying the Eq. (4), the following Eqs. (5) are obtained:

$$
\begin{aligned}
& \Delta \varphi_{12}=2 \pi\left[\left(a_{1} k_{12}+b_{1} M_{12}-a_{0} k_{12}-b_{0} M_{12}\right) h_{1}+\left(a_{2} k_{12}+b_{2} M_{12}-a_{1} k_{12}-b_{1} M_{12}\right) h_{2}\right](5-\mathrm{a}) \\
& \Delta \varphi_{13}=2 \pi\left[\left(a_{1} k_{13}+b_{1} M_{13}-a_{0} k_{13}-b_{0} M_{13}\right) h_{1}+\left(a_{2} k_{13}+b_{2} M_{13}-a_{1} k_{13}-b_{1} M_{13}\right) h_{2}\right](5-\mathrm{b}) \\
& \Delta \varphi_{23}=2 \pi\left[\left(a_{1} k_{23}+b_{1} M_{23}-a_{0} k_{23}-b_{0} M_{23}\right) h_{1}+\left(a_{2} k_{23}+b_{2} M_{23}-a_{1} k_{23}-b_{1} M_{23}\right) h_{2}\right](5-\mathrm{c})
\end{aligned}
$$

Where $k_{i j}=1 / \lambda_{j}-1 / \lambda_{i}, M_{i j}=1 / \lambda_{j}^{3}-1 / \lambda_{i}^{3}$.

There are six variables in the system of Eqs. (5), $a_{1}, a_{2}, h_{1}, h_{2}, b_{1}$, and $b_{2}$, and the others are constants. The environmental RI parameter can be measured before the experiment, so it is considered a known quantity.

Eq. (5-c) minus Eq. (5-b) yields the following Eq. (6):

$$
\frac{\Delta \varphi_{23}}{2 \pi k_{23}}-\frac{\Delta \varphi_{13}}{2 \pi k_{13}}=\left[b_{1}\left(h_{1}-h_{2}\right)+b_{2} h_{2}-b_{0} h_{1}\right]\left(M_{23} / k_{23}-M_{13} / k_{13}\right)
$$

Let us defined $\left(x_{0}, y_{0}\right)$ as the coordinate value at the maximum of the phase distribution, that is $\frac{\partial \varphi(x, y)}{\partial x}=0, \frac{\partial \varphi(x, y)}{\partial y}=0$, from which the interaction between the nucleus and the cytoplasm can be obtained. This point can be regarded as a specific point across the nucleus. The cells were then irradiated with light of wavelength $\lambda_{1}$ along with the Y-axis for phase microscopy imaging, and their phase distribution was gradient-processed to obtain their phase gradient distribution function $\varphi^{\prime}(x, z)$. As shown in Figure 2(b), in the XZ plane of the crossing point $\left(x_{0}, y_{0}\right), h_{1}\left(x_{0}, y_{0}\right)=\Delta z_{1}$ and $h_{2}\left(x_{0}, y_{0}\right)=\Delta z_{2}$.

The nucleus-free region in the phase distribution was selected, i.e., set $\frac{\partial^{2} \varphi(x, y)}{\partial y^{2}}=0$. For $x_{1}$ at any of the nucleus-free regions in Figure 2(c), the position of the extremum $\varphi_{1}\left(x_{1}, y\right)$ was 
found by making $\frac{\partial \varphi_{1}\left(x_{1}, y\right)}{\partial y}=0$ to obtain $y_{1}$. In the XZ plane passing $\left(x_{1}, y_{1}\right), h_{2}\left(x_{1}, y_{1}\right)=0$ was obtained, and the cytoplasm thickness was also obtained by $h_{1}\left(x_{1}, y_{1}\right)=\Delta z_{3}$.

(a)

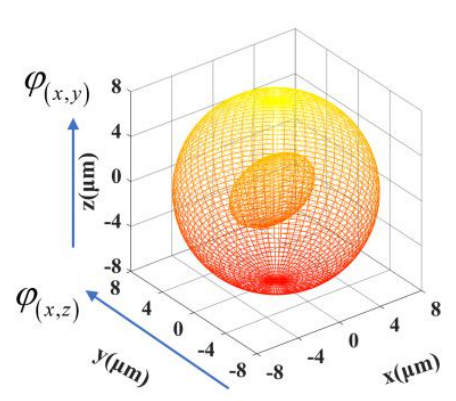

(b)

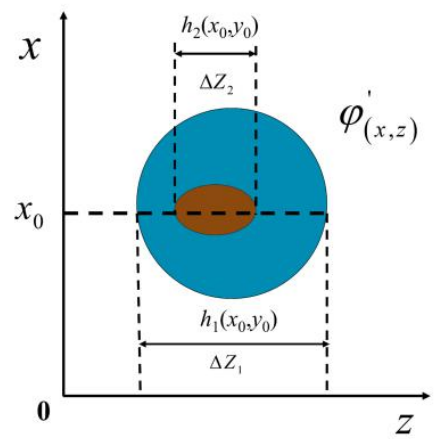

(c)

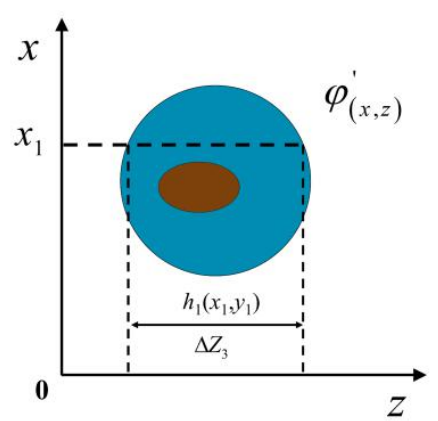

Figure 2. Illustration of the phase gradient analysis. (a) Two-media eccentric ellipsoidal nucleus model; (b) Phase gradient of XOZ plane through the nucleus; (c) Phase gradient of XOZ plane without passing through the nucleus.

Substituting the values of $h_{1}$ and $h_{2}$ at $\left(x_{1}, y_{1}\right)$ into Eqs. (6) and (5-a) to obtain the following equations, respectively:

$$
\begin{gathered}
\frac{\Delta \varphi_{23}\left(x_{1}, y_{1}\right)}{2 \pi k_{23}}-\frac{\Delta \varphi_{13}\left(x_{1}, y_{1}\right)}{2 \pi k_{13}}=\left(b_{1}-b_{0}\right) h_{1}\left(x_{1}, y_{1}\right)\left(M_{23} / k_{23}-M_{13} / k_{13}\right) \\
\Delta \varphi_{12}\left(x_{1}, y_{1}\right)=2 \pi\left[\left(a_{1} k_{12}+b_{1} M_{12}-a_{0} k_{12}-b_{0} M_{12}\right) h_{1}\left(x_{1}, y_{1}\right)\right]
\end{gathered}
$$

From Eq. (7):

$$
b_{1}=\frac{\frac{\Delta \varphi_{23}\left(x_{1}, y_{1}\right)}{2 \pi k_{23}}-\frac{\Delta \varphi_{13}\left(x_{1}, y_{1}\right)}{2 \pi k_{13}}}{\left(M_{23} / k_{23}-M_{13} / k_{13}\right) h_{1}\left(x_{1}, y_{1}\right)}+b_{0}
$$

Substituting $b_{1}$ into Eq. (8), we can get:

$$
a_{1}=\frac{\Delta \varphi_{12}\left(x_{1}, y_{1}\right)}{2 \pi k_{12} h_{1}\left(x_{1}, y_{1}\right)}-\frac{\left(b_{1}-b_{0}\right) M_{12}}{k_{12}}+a_{0}
$$

Similarly, substituting the values of $h_{1}$ and $h_{2}$ at $\left(x_{0}, y_{0}\right)$ into Eq. (6), we get:

$$
\begin{aligned}
\frac{\Delta \varphi_{23}\left(x_{0}, y_{0}\right)}{2 \pi k_{23}}-\frac{\Delta \varphi_{13}\left(x_{0}, y_{0}\right)}{2 \pi k_{13}}= & {\left[b_{1}\left(h_{1}\left(x_{0}, y_{0}\right)-h_{2}\left(x_{0}, y_{0}\right)\right)+b_{2} h_{2}\left(x_{0}, y_{0}\right)-\right.} \\
& \left.b_{0} h_{1}\left(x_{0}, y_{0}\right)\right]\left(M_{23} / k_{23}-M_{13} / k_{13}\right)
\end{aligned}
$$

This results in: 
$b_{2}=\frac{\frac{\Delta \varphi_{23}\left(x_{0}, y_{0}\right)}{2 \pi k_{23}}-\frac{\Delta \varphi_{13}\left(x_{0}, y_{0}\right)}{2 \pi k_{13}}}{\left(M_{23} / k_{23}-M_{13} / k_{13}\right) h_{2}\left(x_{0}, y_{0}\right)}-\frac{\left(h_{1}\left(x_{0}, y_{0}\right)-h_{2}\left(x_{0}, y_{0}\right)\right)}{h_{2}\left(x_{0}, y_{0}\right)} b_{1}+\frac{h_{1}\left(x_{0}, y_{0}\right)}{h_{2}\left(x_{0}, y_{0}\right)} b_{0}$

Substituting $a_{1}, b_{1}$, and $b_{2}$ into Eq. (5-a), we get:

$$
\begin{aligned}
a_{2}= & \frac{\Delta \varphi_{12}\left(x_{0}, y_{0}\right)}{2 \pi k_{12} h_{2}\left(x_{0}, y_{0}\right)}-\frac{a_{2} k_{12}-a_{1} k_{12}-b_{1} M_{12}}{k_{12}}- \\
& \frac{\left(a_{1} k_{12}+b_{1} M_{12}-a_{0} k_{12}-b_{0} M_{12}\right) h_{1}\left(x_{0}, y_{0}\right)}{k_{12} h_{2}\left(x_{0}, y_{0}\right)}
\end{aligned}
$$

Thus, the RI parameters $a_{1}, a_{2}, b_{1}$, and $b_{2}$ of the cytoplasm and nucleus can be obtained from the Eqs. (9-12), and the RI values of cytoplasm and nucleus can be obtained. At last, the decoupling of thickness and RI are realized.

\subsection{Method for determining thickness distribution}

According to Eq. (1), after neglecting the higher terms, it is

$$
n=a+b / \lambda^{2}
$$

After simultaneous Eq. (2) $\varphi_{i}(x, y)=\frac{2 \pi}{\lambda_{i}}\left[\left(n_{1\left(\lambda_{i}\right)}-n_{0\left(\lambda_{i}\right)}\right) h_{1}(x, y)+\left(n_{2\left(\lambda_{i}\right)}-n_{1\left(\lambda_{i}\right)}\right) h_{2}(x, y)\right]$, we can get:

$$
\frac{\varphi_{1}}{k_{1}}=\left(n_{1\left(\lambda_{1}\right)}-n_{0\left(\lambda_{1}\right)}\right) h_{1}+\left(n_{2\left(\lambda_{1}\right)}-n_{1\left(\lambda_{1}\right)}\right) h_{2}
$$

Where $k=\frac{2 \pi}{\lambda}$, thus obtaining:

$$
h_{2}=\frac{1}{n_{2\left(\lambda_{2}\right)}-n_{1\left(\lambda_{2}\right)}}\left[\frac{\varphi_{2}}{k_{2}}-\left(n_{1\left(\lambda_{2}\right)}-n_{0\left(\lambda_{2}\right)}\right) h_{1}\right]
$$

By sorting out Eqs. (14) and (15), we can get:

$$
\frac{\varphi_{1}}{k_{1}}-\frac{\left(n_{2\left(\lambda_{1}\right)}-n_{1\left(\lambda_{1}\right)}\right) \varphi_{2}}{\left(n_{2\left(\lambda_{2}\right)}-n_{1\left(\lambda_{2}\right)}\right) k_{2}}=\frac{\left(n_{1\left(\lambda_{1}\right)}-n_{0\left(\lambda_{1}\right)}\right)\left(n_{2\left(\lambda_{2}\right)}-n_{1\left(\lambda_{2}\right)}\right)-\left(n_{2\left(\lambda_{1}\right)}-n_{1\left(\lambda_{1}\right)}\right)\left(n_{1\left(\lambda_{2}\right)}-n_{0\left(\lambda_{2}\right)}\right)}{n_{2\left(\lambda_{2}\right)}-n_{1\left(\lambda_{2}\right)}} h_{1}
$$

Considering Eq. (3), it is possible to get:

$$
\left(n_{1}-n_{2}\right)_{\lambda_{1}}-\left(n_{1}-n_{2}\right)_{\lambda_{2}}=\left(b_{2}-b_{1}\right) \frac{\lambda_{1}^{2}-\lambda_{2}^{2}}{\lambda_{1}^{2} \lambda_{2}^{2}} \approx 0
$$


Approximate are:

$$
\begin{gathered}
\left(n_{2}-n_{1}\right)_{\lambda_{1}}-\left(n_{2}-n_{1}\right)_{\lambda_{2}}=n_{2}-n_{1} \\
n_{1 \lambda_{1}} n_{2 \lambda_{2}}-n_{2 \lambda_{1}} n_{1 \lambda_{2}}=\left(b_{1} a_{2}-b_{2} a_{1}\right)\left(\frac{\lambda_{2}^{2}-\lambda_{1}^{2}}{\lambda_{1}^{2} \lambda_{2}^{2}}\right) \approx 0
\end{gathered}
$$

By combining Eqs. (16), (18), and (19), we can obtain:

$$
h_{1}(x, y)=\frac{1}{n_{0 \lambda_{2}}-n_{0 \lambda_{1}}}\left(\frac{\varphi_{1}}{k_{1}}-\frac{\varphi_{2}}{k_{2}}\right)
$$

Substituting Eq. (20) into Eq. (2) gives:

$$
h_{2}(x, y)=\frac{1}{n_{2 \lambda_{1}}-n_{1 \lambda_{1}}}\left[\frac{\varphi_{1}}{k_{1}}-\frac{n_{1 \lambda_{1}}-n_{0 \lambda_{1}}}{n_{0 \lambda_{2}}-n_{0 \lambda_{1}}}\left(\frac{\varphi_{1}}{k_{1}}-\frac{\varphi_{2}}{k_{2}}\right)\right]
$$

The thickness distribution of the cell nucleus can be obtained by:

$$
h_{2}(x, y)=\frac{1}{\left(n_{2 \lambda_{1}}-n_{1 \lambda_{1}}\right)\left(n_{0 \lambda_{2}}-n_{0 \lambda_{1}}\right)}\left[\frac{\varphi_{1}}{k_{1}}\left(n_{0 \lambda_{2}}-n_{1 \lambda_{1}}\right)-\frac{\varphi_{2}}{k_{2}}\left(n_{0 \lambda_{1}}-n_{1 \lambda_{1}}\right)\right]
$$

The above calculates the nucleus area. For the non-nucleus area, $n_{2}$ is equal to $n_{1}$, so the above equation does not exist. The distribution of $h_{1}$ can be obtained from Eq. (2), as shown in Eq. (23), while $h_{2}$ is zero.

$$
h_{1}(x, y)=\frac{1}{n_{1 \lambda_{1}}-n_{0 \lambda_{1}}} \frac{\varphi_{1}}{k_{1}}
$$

Whereas the nuclear region can be obtained from the phase gradient distribution of the XY plane (note that the position $h$ is determined from the phase gradient of the XZ plane), and the cytoplasmic thickness distribution of cells can be obtained.

\section{Simulation experiments}

To verify the applicability of the presented theory, a bi-media eccentric ellipsoid nucleocyte model was established as a simulation. The orthogonal bidirectional phase distribution was obtained by quantitative phase microscopy (QPI), and the thickness information was extracted by gradient extraction algorithm to decouple the RI of cells. Figure 2(a) shows a bimedia eccentric ellipsoid nucleocyte model established by MATLAB software, consisting of 
a large sphere and an ellipsoid model. The large sphere simulates the cytoplasm, and the ellipsoid simulates the nucleus. The radius of the large sphere is $8 \mu \mathrm{m}$, and the semi-axis of the ellipsoid nucleus along the $\mathrm{x}-, \mathrm{y}-$, and z-axes is $4 \mu \mathrm{m}, 2.5 \mu \mathrm{m}$, and $3 \mu \mathrm{m}$, respectively, and their central coordinates are $(0,0,0)$ and $(-1,-0.8,0.8)$, respectively. The wavelengths of the light source were set as $632 \mathrm{~nm}, 532 \mathrm{~nm}$, and $488 \mathrm{~nm}$. The cytoplasmic RI were set to 1.3831 , 1.3885 , and 1.3920 at three wavelengths, respectively, and likewise, the nuclear refractive indexes were set at $1.4228,1.4281$, and 1.4315 . In addition, the ambient liquid RI were set to $1.3419,1.3468$, and 1.3500 . The size of the information receiving screen is set to $20 \times 20 \mu \mathrm{m}$ and the size of the interference image is set to $500 \times 500$ pixels. Therefore, the distance between two adjacent pixels on the recording plane is $20 / 500 \mu \mathrm{m}$. Considering the influence of noise in the actual experiment, $35 \mathrm{~dB}$ Gaussian white noise was added to the simulation.

According to the above parameter setting and the above method, phase imaging simulation was performed based on MATLAB. Figure 3 shows the 3D and 2D phase distribution in the z-axis direction at 632nm (a-b), 532nm (c-d), and 488nm (e-f). Figure 3(b, d, f) shows a convex structure representing the nucleus and preliminary determination of the nuclei number and size. In Figure 3(b, d ,f), a protruding structure representing the nucleus can be clearly seen, which can be used to determine the number and size of nuclei preliminarily. Figure $3(\mathrm{~g}-\mathrm{h})$ shows the $3 \mathrm{D}$ and $2 \mathrm{D}$ phase distributions in the $\mathrm{y}$-axis direction at $632 \mathrm{~nm}$.

(a)

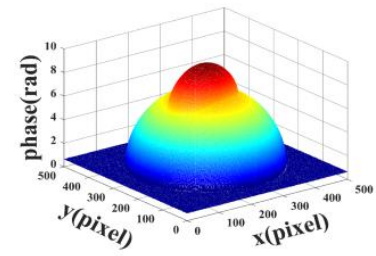

(e)

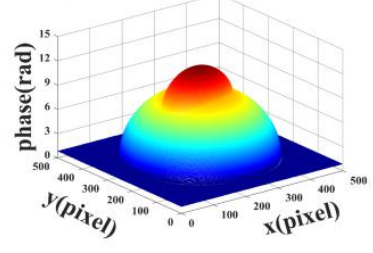

(b)

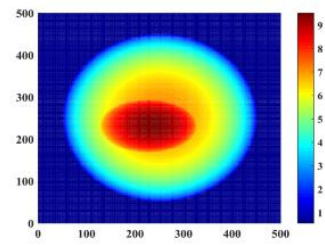

(f)

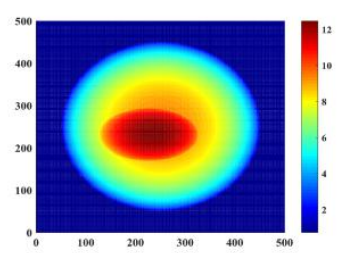

(c)

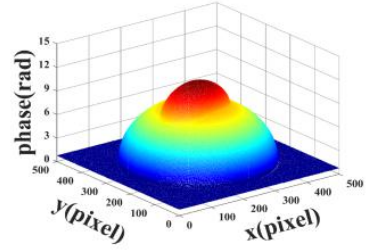

$(\mathrm{g})$

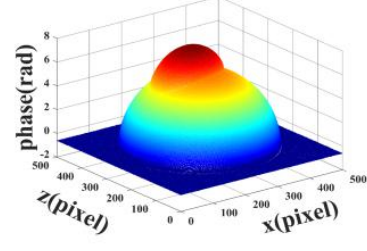

(d)

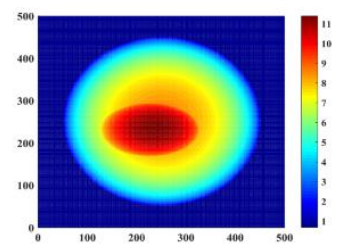

(h)

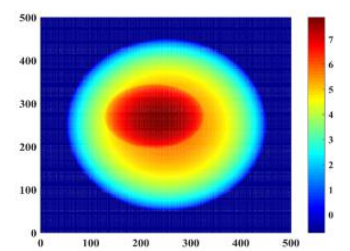

Figure 3. Phases of simulation experiment. (a) and (b) are 3D and 2D phases in z-axis direction at 632nm, (c) and (d) are 3D and 2D phases in the z-axis direction at $532 \mathrm{~nm}$, (e) and (f) are $3 D$ and $2 D$ phase in the $\mathrm{z}$-axis direction at 488 $\mathrm{nm},(\mathrm{g})$ and $(\mathrm{h})$ are $3 \mathrm{D}$ and $2 \mathrm{D}$ phases in the $\mathrm{y}$-axis direction at $632 \mathrm{~nm}$ 
After the phase distribution of orthogonal bidirectional is obtained, the edge information of the phase image is extracted by a gradient extraction algorithm. For brevity, only the phase distribution shown in Figure $3(\mathrm{~g})$ demonstrates the gradient along the $\mathrm{x}$-direction, and the obtained gradient image is shown in Figure 4(a). Figure 4(b-c) corresponds to the phase gradient curve on the AD section and the EF section, respectively in Figure 4(a).

(a)

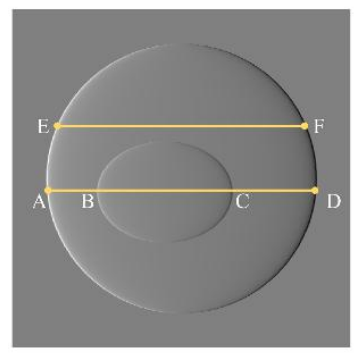

(b)

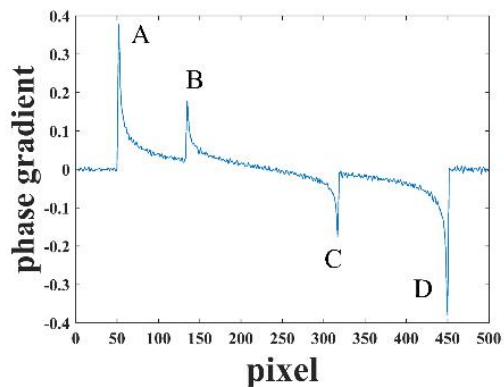

(c)

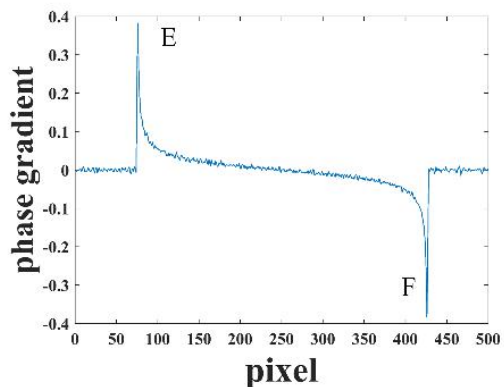

Figure 4. Gradient analysis. (a) phase gradient distribution in Y direction at 632nm; (b) phase gradient curve on AD section corresponding to (a); (c) gradient curve on EF section corresponding to (a).

According to the gradient distribution, the thicknesses $h_{1}\left(x_{0}, y_{0}\right)$ and $h_{2}\left(x_{0}, y_{0}\right)$ passing through the nucleus are calculated. A phase gradient change curve is selected along the AD direction in the gradient distribution diagram. Figure 4(b) shows that the curve passes through the environment fluid, cytoplasm, nucleus, cytoplasm, and finally back to the surrounding fluid. Thus, there are four changes in the RI: $n_{0} \rightarrow n_{1} \rightarrow n_{2} \rightarrow n_{1} \rightarrow n_{0}$, there are four jumps. According to the concept of forwarding difference (latter term minus former term), the phase change depends on the change in RI when the cell is divided into equalthickness samples along the optical axis. In the simulation, $n_{0}<n_{1}$, the first and second jump points are positive, and $n_{1}<n_{2}$ in the third and fourth jump points are negative. These four jump points correspond to the ABCD points in the diagram, and their coordinate positions are $52,135,317$, and 450 pixels. By calculating the distance between point $\mathrm{A}$ and point $\mathrm{D}$ on the curve, the thickness $h_{1}\left(x_{0}, y_{0}\right)$ can be obtained, its value is 398 pixels $(15.952 \mu \mathrm{m})$, and the error is $0.048 \mu \mathrm{m}$. The distance between point $\mathrm{B}$ and point $\mathrm{C}$ on the curve is calculated to obtain the thickness value $h_{2}\left(x_{0}, y_{0}\right)=182$ pixels $(7.2950 \mu \mathrm{m})$, and the error is $0.0705 \mu \mathrm{m}$.

Similarly, the thickness $h_{1}\left(x_{1}, y_{1}\right)$ of the uncrossed nucleus is calculated from the gradient distribution. A phase gradient variation curve along the EF direction was selected in the gradient distribution diagram, as shown in Figure 4(c), which passes through the ambient 
fluid, cytoplasm, cytoplasm, and finally back to the ambient fluid. Thus, there are two kinds of changes in the RI: in $n_{0} \rightarrow n_{1} \rightarrow n_{1} \rightarrow n_{0}$, there are two jumps, the first jump point is positive, and the second jump point is negative. The coordinate positions of these two jump points were 76 and 426 pixels. The calculated distance between $\mathrm{E}$ and $\mathrm{F}$ points on the curve to obtain the thickness $h_{1}\left(x_{1}, y_{1}\right)$ was 350 pixels $(14.028 \mu \mathrm{m})$.

However, before calculating $h_{1}\left(x_{0}, y_{0}\right), h_{2}\left(x_{0}, y_{0}\right)$ and $h_{1}\left(x_{1}, y_{1}\right)$, it is necessary to determine the coordinate value $\left(x_{0}, y_{0}\right)$ at the maximum value of the phase distribution. Taking the phase distribution in the Z-axis direction at $632.8 \mathrm{~nm}$ as an example, $x_{0}=241$ pixels, $y_{0}=239$ pixels obtained by MATLAB calculation; similarly, $\mathrm{x}_{1}=346$ pixels, $\mathrm{y}_{1}=259$ pixels are obtained.

In the experiment, the RI of the ambient liquid is known or measurable. According to the method in this paper, the cytoplasmic RI $n_{1}$ can be calculated according to formulas (9), (10), and (13). The RI $n_{2}$ of the nucleus can be calculated according to formulas (11), (12), and (13). Eqs. (22) and (23) calculated the thickness distribution of cytoplasm and nucleus under the cell model, respectively. Figure 5(a-b) shows the distribution of the cytoplasm thickness and the distribution of the nucleus thickness under the two-media eccentric ellipsoid cell model. The thickness distribution reflects the spheroidal shape of the cytoplasm and the ellipsoid shape of the nucleus. Thus, the thickness error analysis was performed. Figure 6(a,c) represents the theoretical thickness distributions of the cytoplasm and nucleus, and the $3 \mathrm{D}$ error distribution can be obtained by subtracting them from the simulated thickness distributions in Figure 5(a, b), respectively. Figure 6(b) shows that the floating range of cytoplasm thickness error is concentrated between $-1 \mu \mathrm{m}$ and $1 \mu \mathrm{m}$, with the maximum error not exceeding $1.5 \mu \mathrm{m}$ and the maximum relative error being $2.24 \%$. Figure $6(\mathrm{~d})$ shows that the floating range of cell nuclear thickness error is mainly between $-0.4 \mu \mathrm{m}$ and $0.4 \mu \mathrm{m}$, the maximum error is not more than $0.5 \mu \mathrm{m}$, and the maximum relative error is $5.37 \%$. 
(a)

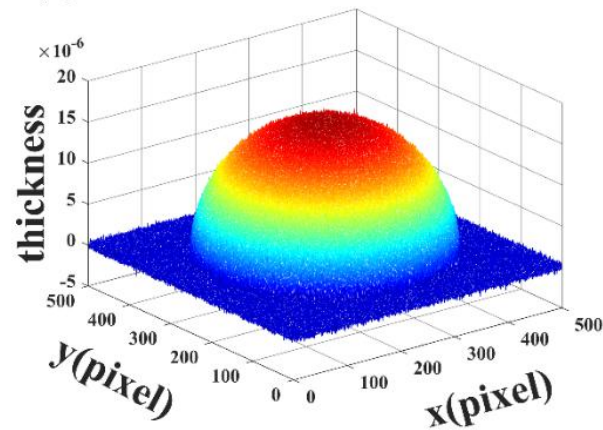

(b)

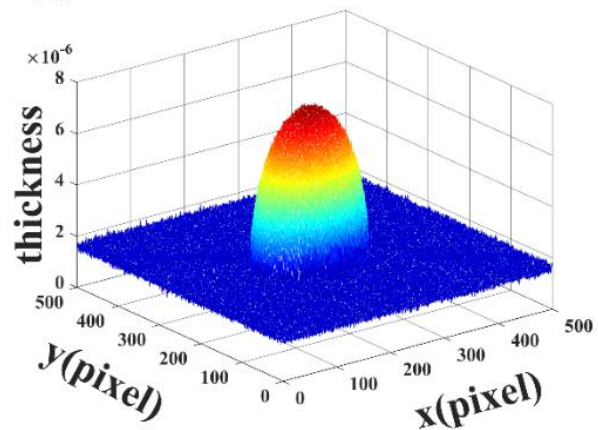

Figure 5. Simulated thickness results: substructure thickness distribution under the two-media eccentric ellipsoidal nucleus cell model. (a) cytoplasmic thickness distribution; (b) nucleus thickness distribution.

(a)

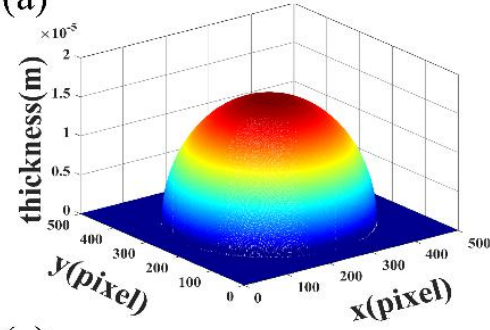

(c)

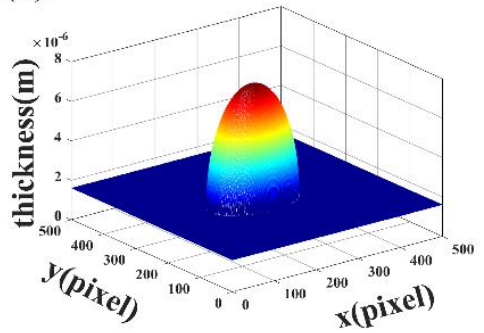

(b)

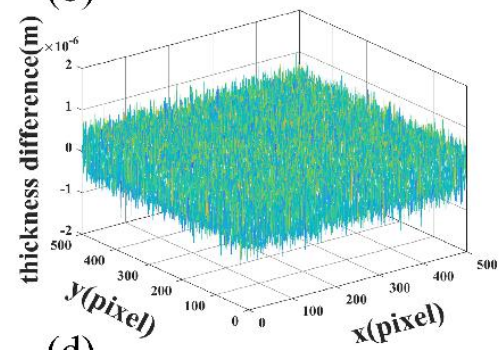

(d)

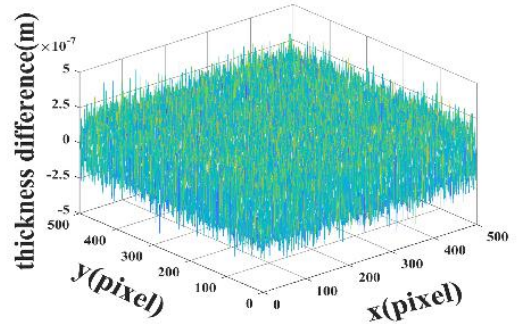

Figure 6. Thickness error analysis results. (a) cytoplasm theoretical thickness; (b) 3D error distribution between cytoplasm theoretical thickness and simulation thickness; (c) nucleus theoretical thickness; (d) 3D error distribution between nucleus theoretical thickness and simulation thickness.

Table 1. Refractive index of the bi-media eccentric ellipsoidal core model.

\begin{tabular}{|c|c|c|c|}
\hline Wavelength(nm) & 632.8 & 532 & 488 \\
\hline Cytoplasm RI Set value $n_{1}$ & 1.3831 & 1.3885 & 1.3920 \\
\hline Cytoplasm RI Solution value $n_{1}$ & 1.3846 & 1.3900 & 1.3935 \\
\hline Cytoplasm RI Relative error & $0.11 \%$ & $0.11 \%$ & $0.11 \%$ \\
\hline Nucleus RI Set value $n_{2}$ & 1.4228 & 1.4281 & 1.4315 \\
\hline Nucleus RI Solution value $n_{2}$ & 1.5188 & 1.5027 & 1.4923 \\
\hline Nucleus RI Relative error & $6.75 \%$ & $5.22 \%$ & $4.25 \%$ \\
\hline
\end{tabular}




\section{Conclusions}

Since cell sub-structure morphology is closely related to cell RI distribution, and RI is coupled with its thickness parameter in phase microscopy, the decoupling of RI and thickness has been a research topic. The traditional decoupling method of RI and thickness have not yet provided a good solution for decoupling RI and thickness of multi-media, non-spherical cell. In this paper, we propose a decoupling method for three-wavelength phase microscopy, that can effectively remedy the shortcomings of the traditional decoupling methods. The method only requires neglecting the high-order dispersion of the medium, as the effect of high-order dispersion is two orders of magnitude less than primary dispersion. The method in this paper ignores the medium's high-order dispersion because this hypothesis is effectively in general case.

The decoupling method in this paper does not require cell morphology, so this method is suitable for cells of any shape and structure. Therefore, it is particularly effective for blood cells. The experimental simulation results show that the error of RI and thickness of cytoplasm is less than $1 \%$, and the error of nucleus is about $5 \%$, demonstrating this method's accuracy. Further research of the decoupling methods of RI and thickness for multinucleated cells in different media is required.

\section{Acknowledgments}

This work was supported by National Natural Science Foundation of China (No.11874184), Postdoctoral Science Foundation of China (No. 2019M651769); Postdoctoral Science Foundation of Jiangsu Province (No. 2018K032A); Training Program Foundation of Young Talents of Jiangsu University (No.411190007); The 19th Batch of College Students Research Projects (No.Y19A051).

\section{References}

[1] G. Dardikman, N.T. Shaked, Review on methods of solving the refractive indexthickness coupling problem in digital holographic microscopy of biological cells, Optics Communications. 422 (2018) 8-16. https://doi.org/10.1016/j.optcom.2017.11.084. 
[2] N. Lue, G. Popescu, T. Ikeda, R. R. Dasari, K. Badizadegan,M. S. Feld, Live cell refractometry using microfluidic devices, Opt. Lett. 31 (2006) 2759-2761. https://doi.org/10.1364/OL.31.002759.

[3] B. Kemper, S.Kosmeier, P. Langehanenberg,G. von Bally, I. Bredebusch, W. Domschke, J. Schnekenburger, Integral refractive index determination of living suspension cells by multifocus digital holographic phase contrast microscopy, J. Biomed. Opt. 12 (2007) 054009. https://doi.org/10.1117/1.2798639.

[4] P. Eaton, P. West, Atomic force microscopy, Oxford University Press, 2010.

[5] J Pawley. Handbook of biological confocal microscopy, Vol. 236, Springer Science \& Business Media, 2006.

[6] C. L. Curl, C. J Bellair, T. Harris, B. E. Allman, P. J. Harris, A. G. Stewart,et al,Refractive index measurement in viable cells using quantitative phase-amplitude microscopy and confocal microscopy, Cytometry Part A: The Journal of the International Society for Analytical Cytology. 65 (2005) 88-92. https://doi.org/10.1002/cyto.a.20134.

[7] A. Kuś, M. Dudek, B. Kemper, M. Kujawińska, and A. Vollmer, Tomographic phase microscopy of living three-dimensional cell cultures, J. Biomed. Opt. 19 (2014) 046009. https://doi.org/10.1117/1.JBO.19.4.046009.

[8] M. Habaza, B. Gilboa, Y. Roichman, and N. T. Shaked. Tomographic phase microscopy with $180^{\circ}$ rotation of live cells in suspension by holographic optical tweezers, Opt. Lett. 40 (2015) 1881-1884. https://doi.org/10.1364/OL.40.001881.

[9] D. Jin, R. Zhou, Z. Yaqoob, and Peter T. C. So. Tomographic phase microscopy: principles and applications in bioimaging [Invited], J. Opt.Soc. Am. B. 34 (2017) B64. https://doi.org/10.1364/JOSAB.34.000B64.

[10] G. Dardikman, Y. N. Nygate, I. Barnea, N. A. Turko, G. Singh, B. Javidi, and N. T. Shaked, Integral refractive index imaging of flowing cell nuclei using quantitative phase microscopy combined with fluorescence microscopy, Biomed. Opt. Express. 9 (2018) 1177-1189. https://doi.org/10.1364/BOE.9.001177.

[11] G. Dardikman, G. Singh, N. T. Shaked, Four dimensional phase unwrapping of dynamic objects in digital holography, Opt. Express. $26 \quad$ (2018) 3772-3778. https://doi.org/10.1364/OE.26.003772.

[12]N. Cardenas, S. Mohanty, Decoupling of geometric thickness and refractive index in quantitative phase microscopy, Opt. Lett. $38 \quad$ (2013) 1007-1009. https://doi.org/10.1364/OL.38.001007. 
[13] B. Rappaz, A. Barbul, Y. Emery, R. Korenstein, C. Depeursinge, P. J. Magistretti, P. Marquet, Comparative study of human erythrocytes by digital holographic microscopy, confocal microscopy, and impedance volume analyzer, Cytometry Part A: The Journal of the International Society for Analytical Cytology. $73 \quad$ (2008) 895-903. https://doi.org/10.1002/cyto.a.20605.

[14] B. Rappaz, F. Charrière, C. Depeursinge, P. J. Magistretti, P. Marquet, Simultaneous cell morphometry and refractive index measurement with dual-wavelength digital holographic microscopy and dye-enhanced dispersion of perfusion medium, Opt. Lett. 33 (2008) 744-746. https://doi.org/10.1364/OL.33.000744.

[15] B. Rappaz, P. Marquet, E. Cuche, Y. Emery, C. Depeursinge, and P. J. Magistretti, Measurement of the integral refractive index and dynamic cell morphometry of living cells with digital holographic microscopy, Opt. Express. 13 (2005) 9361-9373. https://doi.org/10.1364/OPEX.13.009361.

[16] D. Boss, J. Kühn, P. Jourdain, C. D. Depeursinge, P. J. Magistretti, P.P. Marquet M.D, Measurement of absolute cell volume, osmotic membrane water permeability, and refractive index of transmembrane water and solute flux by digital holographic $\begin{array}{lllllll}\text { microscopy, } & \text { J. } & \text { Biomed. } & \text { Opt. } & 18 & \text { (2013) } & 036007 .\end{array}$ https://doi.org/10.1117/1.JBO.18.3.036007.

[17] M. R. Jafarfard, S. Moon, B. Tayebi, D. Y. Kim, Dual-wavelength diffraction phase microscopy for simultaneous measurement of refractive index and thickness, Opt. Lett. 39 (2014) 2908-2911. https://doi.org/10.1364/OL.39.002908.

[18] Z. D. Xin, Y. Y. Xu, Y. Ji, W. F. Jin, H. R. Zheng, L. Zhang, Y. W. Wang, The homogeneous and dual-medium cell's refractive index decoupling method and entropy tomographic imaging, High Power Lasers, High Energy Lasers, and Silicon-Based Photonic Integration. 101520T (2016). https://doi.org/10.1117/12.2246701.

[19] Guangwei Peng, Research on Refractive Index Decoupling Method of Multimedia Cells Based on Quantitative Phase Micromorphology Reconstruction, Jiangsu University, 2020. https://doi.org/10.27170/d.cnki.gjsuu.2020.002058. 